\title{
Seguimiento de pacientes dados de alta de un hospital de día para adolescentes. Estudio descriptivo
}

RESUMEN: Estudio descriptivo del Hospital de Día para Adolescentes de Gavà (Barcelona) a partir de una encuesta ad hoc que se administró al ingreso, al alta y, posteriormente, a los seis meses y al año.

PALABRAS CLAVE: Adolescencia, hospital de día, valoración subjetiva, seguimiento.
SUMMARY: Descriptive study in the adolescent day-hospital of Gavà (Barcelona) is based on an ad hoc questionnaire given at the time of income, discharge, six months after discharge and finally one year after discharge.

KEY WORDS: Adolescence, day-hospital, subjective appraisal, follow-up.

Si bien los hospitales de día, como «dispositivos intermedios», tienen una larga tradición en la atención a los pacientes adultos con trastornos mentales $(1 ; 2)$, en cuanto a la población infantil y juvenil, el surgimiento de este tipo de servicios en la asistencia pública es relativamente reciente y se ha hecho, en muchos casos, desde una perspectiva comunitaria $(3 ; 4)$.

Por lo que a nuestro país se refiere y a excepción de algunas experiencias concretas, como las unidades de atención psiquiátrica para niños y adolescentes en hospitales generales o pediátricos ${ }^{1}$, solo en la segunda mitad de los años ochenta se empiezan a crear los hospitales de día psiquiátricos para niños y, más tarde, específicos para adolescentes. «Los actuales dispositivos clínicos -dice A. Jiménez- para el abordaje de la patología mental grave en infancia y adolescencia que existen en las distintas comunidades autónomas parten en muchos casos de diferentes formas de organización y de ubicación, perteneciendo además a diferentes organismos, siendo sus características las del hospital de día y lo común la terapia institucional» (5).

Conviene mencionar, asimismo, que junto con la progresiva puesta en marcha de los hospitales de día a escala estatal, en los últimos años se ha iniciado un proceso que reclama una definición y estructuración «clara y suficiente» de una red asistencial de salud mental infanto-juvenil. Propone que ha de incluir, además de la atención ambulatoria y los dispositivos intermedios (de hospitalización parcial), los dispositivos de internamiento terapéutico y residencial (unidades de hospitalización breve, de media y larga estancia y los dispositivos

El primer hospital de Día para niños y adolescentes se creó hace poco más de 10 años en el Hospital Infantil de San Juan de Dios de Barcelona. Se trataba de una unidad que, a pesar de funcionar de forma menos asilar, sin embargo, no dejaba de estar dentro -y no sólo físicamente- del «ámbito» hospitalario. 
socio-educativos-sanitarios) para aquellos niños con patología mental grave, especialmente los trastornos de conducta de diversa etiología (6).

En Cataluña ${ }^{2,3}$, el primer hospital de día para adolescentes, sectorizado e integrado en la comunidad, inició su actividad a finales de 1998 en el municipio de Gavà. Se trata de una unidad asistencial de hospitalización parcial que combina el tratamiento individual, grupal y familiar, con recursos psicoterapéuticos, ocupacionales, farmacológicos e institucionales. Atiende a chicos y chicas entre 12 y 18 años que presentan trastornos psicopatológicos o disarmonías evolutivas graves (inicio de anorexia, fobias escolares, inhibiciones graves, etc.; en definitiva, «crisis adolescente»). No son candidatos a ingreso en el centro los casos de conductas suicidas o parasuicidas, retraso mental moderado o grave, así como los problemas de drogadicción no incipiente. El equipo asistencial lo forman 2 médicos psiquiatras, 2 psicólogos, 6 educadores, 1 enfermera y 1 trabajador social. El Hospital de día de Gavà pertenece a la Fundación Orienta, entidad proveedora de servicios de salud mental de la Generalitat de Catalunya, y en la actualidad dispone de 36 plazas concertadas.

Este centro, igual que los demás que integran la red de hospitales de día de Cataluña ${ }^{4}$, cuentan con un docente que, integrado en el equipo asistencial, realiza tareas educativas adaptadas a la situación clínica de los adolescentes ingresados. De esta forma, además de mantener -o favorecer- el grado de escolarización de los pacientes, evita una desvinculación radical de la que hasta el momento ha sido su «vida escolar». Más aun, hablando de este aspecto concreto, Veziat (7) dice: «puede y debe existir un método de trabajo tradicional en un establecimiento como el hospital de día. Sin embargo, más allá del método, la aceptación incondicional de la personalidad del niño, de sus posibilidades escolares, se asocia a un material escolar accesible, siempre dispuesto, abierto. Esto no es sólo una hipótesis de trabajo, es una opción personal en lo que se refiere al futuro escolar y psicológico del niño».

En relación con el trabajo que presentamos lo que nos animó a realizarlo, fue, entre otros motivos, su reciente puesta en marcha y la falta de estudios en nuestro medio con el principal objetivo de conocer la evolución de los pacientes dados de

\footnotetext{
2 El Hospital de Día para Adolescentes de Gavà, a diferencia de los anteriores equipamientos, plantea su actuación de forma más comunitaria y vinculada con el entorno familiar, social y educativo del adolescente. Esta línea fue, desde los inicios, una apuesta firme de la Fundación Orienta.

3 El artículo de Ana Jiménez, citado anteriormente y publicado en el año 2001, al final presenta una relación de participantes en el grupo de trabajo sobre Hospital de Día Psiquiátrico para niños y adolescentes. Pues bien, de los 25 profesionales que representan diferentes hospitales de día, 18 provienen de Cataluña, 2 de Pamplona, 1 de Las Palmas de Gran Canarias, 2 de Madrid, 1 de Fuenlabrada y 1 de Alcázar de San Juan.

4 En la actualidad en Cataluña están en funcionamiento 13 hospitales de día para adolescentes, de los cuales 10 están en la provincia de Barcelona, 1 en la de Girona, 1 en Lleida y 1 en Tarragona.
} 
alta. Pues bien, los aspectos que inicialmente veíamos como un aliciente, al final resultaron ser un verdadero obstáculo, especialmente, por la escasa literatura encontrada y los pocos estudios de seguimiento publicados hasta ahora ${ }^{5}$. Se trata, pues, de una primera aproximación que, más allá de las limitaciones metodológicas y del corto tiempo estudiado, viene a confirmar algunas impresiones sobre la evolución clínica y vital de estos chicos y chicas, tal como se apunta en las conclusiones.

\section{Material y Método}

Formaron parte del estudio todos los pacientes que ingresaron entre enero de 2001 y diciembre de 2002 y que fueron dados de alta clínica en este mismo periodo. Puesto que se trataba de conocer la evolución, una vez finalizado el proceso terapéutico en el hospital de día, las altas por otros motivos (abandonos, traslados, altas voluntarias, etc.) se descartaron. Para la obtención de los datos se utilizó una encuesta ad hoc, estructurada a partir de tres tipos de variables: pragmáticas -situación familiar, laboral, diagnóstico, tratamiento, e ingresos- subjetivas -valoración del estado anímico por parte del paciente o la familia- y sociodemográficas -edad y sexo-. En total el cuestionario estaba formado por 19 preguntas con puntuaciones según la escala de Likert (1-5). En relación con el instrumento utilizado, destacar que si bien las encuestas o escalas validadas aportan una mayor «confianza» estadística, la elección por nuestra parte de «variables pragmáticas» obedeció, después de diversas discusiones, a la necesidad de tener un elemento tangible sobre el estado clínico del paciente: reingreso después del alta, aumento de la medicación, agudización de la sintomatología, etc. Tiempo después de acabar el trabajo vimos con sorpresa que este tipo de variables tienen cada vez mayor presencia en estudios sobre eficacia de determinados psicofármacos (8). En definitiva, pues, se trata de un estudio descriptivo longitudinal, de un año de seguimiento.

Para la recogida de los datos se utilizó básicamente el mismo tipo de cuestionario, realizada en cuatro momentos diferentes: al ingreso del paciente en el hospital de día, en el momento del alta clínica, a los seis meses y al año de haberse marchado del centro. De los 81 pacientes dados de alta clínica y que constituían la muestra total, 48 contestaron la encuesta al ingresar, al alta, a los

Estudios de seguimiento propiamente dicho encontramos muy pocos, una revisión más amplia nos permitió recabar información sobre estudios relacionados con modalidades terapéuticas (10), dificultades de contención en el niño (11) y un estudio sobre la satisfacción de pacientes y familias en servicios de hospitalización parcial (12). 
6 meses y 37 cumplieron finalmente con el calendario previsto. En este sentido señalar que una de las dificultades de los estudios de seguimiento es, precisamente, poder contactar con los chicos y chicas una vez que han abandonado el hospital debido, sobre todo, a cambios de teléfono, de domicilio, etc. Frente a estas dificultades, señalar que tanto los adolescentes como las familias valoraron positivamente el esfuerzo por conocer su estado después del alta y no como una intromisión, como en un principio habíamos previsto. En el trabajo de campo participaron diversas personas, siendo el trabajador social quien finalmente se hizo cargo de pasar las encuestas: dos mediante entrevista directa -cuando el paciente estaba en el centro- y dos por consulta telefónica -al cabo de seis meses y al año-.

Para el procesamiento de la información se elaboró una base de datos a partir de las respuestas obtenidas, a la cual se añadieron otros datos que ya disponíamos (sexo, edad, diagnóstico clínico). En algunas variables como el diagnóstico o preguntas abiertas se realizan reagrupaciones en categorías (como las establecidas por la CIE-10, para los diagnósticos). Para el análisis de los datos se establecieron comparaciones sobre la evolución del paciente a lo largo del ingreso, alta, 6 meses y al año, a partir de las frecuencias encontradas.

\section{Resultados}

\section{Variables sociodemográficas}

La muestra estaba formada mayoritariamente por hombres (69\%). La media de edades se situaba en 15 años, siendo la moda de 17 años. Diferenciando las edades según sexo, se observa una diferencia importante entre hombres y mujeres en la moda. En los varones, la frecuencia más elevada se da en edades inferiores ( moda $=15$ ), a diferencia de las mujeres en las que la frecuencia más elevada se da alrededor de los 17 años. Sin embargo, las medias no difieren tanto (15,1 en hombres y 15,9 en mujeres).

\section{Variables pragmáticas}

A. Farmacología: Aproximadamente un $60 \%$ de los adolescentes que ingresaron en el hospital de día seguían un tratamiento farmacológico, y casi un $20 \%$ tomaban más de un fármaco. En el momento del alta clínica un 33\% seguían tratamiento farmacológico y sólo un $0,5 \%$ de los pacientes obtiene el alta con prescripción de más de un fármaco. A los 6 meses, el 31\% continúa en tratamiento farmacológico, mientras que al cabo de un año esta cifra pasa a ser casi el 21,6\% (Tabla 1). 
ORIGINALES Y REVISIONES

Tabla 1

Pacientes del estudio en tratamiento farmacológico. Números absolutos y porcentajes

\begin{tabular}{lcccc}
\hline & Con fármacos & Sin fármacos & T & (\% con medicación) \\
\hline Ingreso & 27 & 21 & 48 & 56,3 \\
\hline Alta & 16 & 32 & 48 & 33,3 \\
\hline A los 6 meses del alta & 15 & 33 & 48 & 31,3 \\
\hline Al año del alta & 8 & 29 & 37 & 21,6 \\
\hline
\end{tabular}

El tipo de medicamentos más utilizados, durante la estancia en el hospital como a lo largo de la consulta, fueron mayoritariamente los antipsicóticos (risperidona, olanzapina, haloperidol y clozapina -sólo un paciente-), seguido de antidepresivos y ansiolíticos.

B. Ámbito familiar: en relación con el núcleo de convivencia, la situación familiar y de convivencia se mantiene, no hay cambios significativos en las familias de la muestra evaluada. Casi el $70 \%$ de la muestra de pacientes convive con la familia nuclear y un $25 \%$ residen con uno de los padres en una estructura familiar monoparental.

C. Ámbito estudiantil y laboral: se observó un aumento progresivo de los chicos y chicas que trabajan (de ninguno en el ingreso hasta el 27\% al año del alta) y una disminución en el campo «sólo estudia». Este hecho se podría explicar en parte por un factor temporal del crecimiento de los chicos. Sin embargo, un dato importante es el del grupo que no trabaja ni estudia. Al ingreso, un $25 \%$ de la muestra evaluada se encuentra en este grupo, pero en el momento del alta y a los 6 meses de valoración el porcentaje se reduce al 18\% de los jóvenes inactivos. Al año del alta hospitalaria sólo un $16 \%$ de los chicos y chicas no estudian ni trabajan (Tablas 2 y 3 ).

Tabla 2

Tipo de relaciones. Porcentajes

\begin{tabular}{lcccc}
\hline & Ingreso & Alta & 6 meses & 1 año \\
\hline Buenas o muy buenas & 56,3 & 60,4 & 62,5 & 67,6 \\
\hline Regulares & 31,3 & 33,3 & 31,3 & 21,6 \\
\hline Conflictivas o muy conflictivas & 12,5 & 4,2 & 4,2 & 8,1 \\
\hline No consta & 0,0 & 2,1 & 2,1 & 2,7 \\
\hline TOTAL & 100 & 100 & 100 & 100 \\
\hline
\end{tabular}


Tabla 3

Situación escolar y laboral de los pacientes. Porcentajes

\begin{tabular}{lrrrr}
\hline & Ingreso & Alta & 6 meses & 1 año \\
\hline No trabaja ni estudia & 25,0 & 18,8 & 18,8 & 16,2 \\
\hline Sólo estudia & 68,8 & 68,8 & 64,6 & 51,4 \\
\hline Sólo trabaja & 0,0 & 6,3 & 10,4 & 27,0 \\
\hline Ni trabaja ni estudia & 2,1 & 6,3 & 4,2 & 2,7 \\
\hline No contesta & 4,2 & 0,0 & 2,1 & 2,7 \\
\hline TOTAL & 100 & 100 & 100 & 100 \\
\hline
\end{tabular}

D. Tratamientos posteriores y continuidad asistencial en la red de salud mental: A los 6 meses, el 10\% ha asistido a algún servicio de la red de salud mental. El $31 \%$ siguen visitas periódicas en el CSMIJ/CSMA de referencia, pasando al $24 \%$ al año del alta.

Un $18 \%$ sigue un tratamiento individual o grupal a los 6 meses, y un $24 \%$ lo hace al año de obtener el alta del servicio.

Mejoras subjetivas

Las causas o motivos por los que se solicitó el ingreso en el hospital de día fueron, principalmente, por auto y/o heteroagresividad, problemas conductuales, aislamiento y sintomatología depresiva.

Esta clínica coincide, en general, con los diagnósticos más frecuentes de la muestra que fueron los de psicosis (11 pacientes) y trastornos de conducta (11 pacientes).

En el ámbito relacional se puede observar cómo la calidad de las relaciones consideradas regulares y conflictivas disminuyen para dar paso a un aumento en las relaciones buenas o muy buenas. Este aumento de la satisfacción en las relaciones de convivencia se detecta a los 6 meses y se mantiene al año de alta del Hospital de Día (Gráfico 1). 


\section{Gráfico 1}

Calidad de las relaciones de convivencia. Porcentajes.

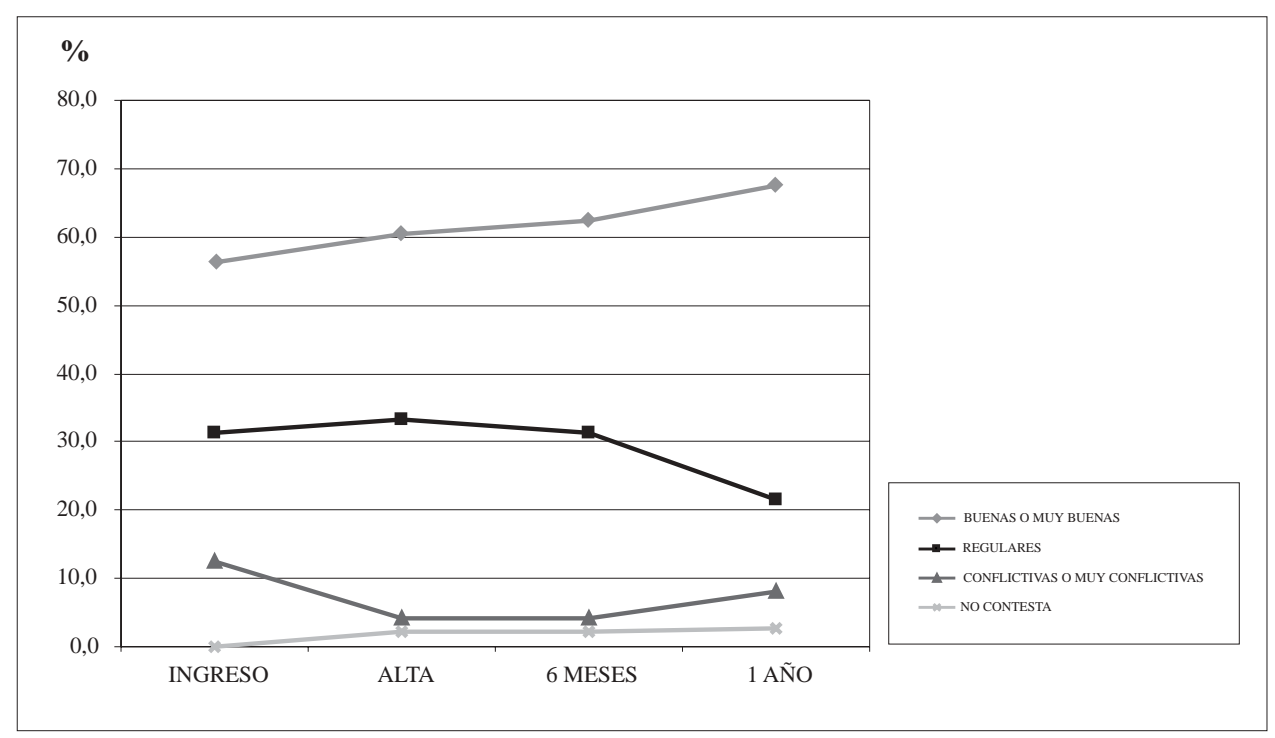

De las respuestas obtenidas a partir de la visión de los pacientes, obtenemos que en el momento del alta, un $15 \%$ considera que han desaparecido los motivos por los cuales había ingresado. A los 6 meses y al año, el 50\% de los chicos consideran que han remitido totalmente sus síntomas, y sólo un caso considera que ha empeorado a los 6 meses. Un 8\% no nota cambio alguno ni en el alta, tampoco a los 6 meses ni al año de ser dado de alta clínica del Hospital de Día (Tabla 4).

Tabla 4

Valoración subjetiva de la situación clínica post alta. Porcentajes

\begin{tabular}{lccc}
\hline \multicolumn{1}{c}{ Los motivos por los cuales va ingresar } & Alta & 6 meses & 1 año \\
\hline Han desaparecido (Remisión del cuadro) & 14,6 & 47,9 & 51,4 \\
\hline Han mejorado (Clínicamente) & 72,9 & 39,6 & 37,8 \\
\hline Continúan igual & 8,3 & 8,3 & 8,1 \\
\hline Ha empeorado & 0,0 & 2,1 & 0,0 \\
\hline No contesta & 4,2 & 2,1 & 2,7 \\
\hline TOTAL & 100 & 100 & 100
\end{tabular}




\section{Discusión}

Una primera cuestión a destacar, desde un punto de vista más general, es un cierto desajuste entre el esfuerzo realizado y los resultados obtenidos. Las razones -a menudo inevitables, cuando se trata de la investigación- tienen que ver, en nuestro caso, con dificultades metodológicas y prácticas. Pues bien, con el fin de paliar la falta de un instrumento validado para la recogida de información, se planteó seguir la evolución clínica del paciente, a partir de aspectos «tangibles»-cómo mantenían la medicación, nuevos brotes o crisis que requieran ingresos hospitalarios, aislamiento domiciliario, etc.-; en definitiva, a través de variables pragmáticas -como se vienen denominando últimamente-. El intento por obtener este tipo de respuestas, tampoco dio el resultado esperado, sobre todo por las dificultades para contactar con los pacientes una vez que se marchan del hospital. Esta situación, habitual en los estudios de seguimiento, tiende a acentuarse en periodos de poca estabilidad -como sin duda es la adolescencia- de los sujetos que forman parte del estudio.

Junto con lo anterior señalar que si bien el trabajo aporta unos datos sobre la evolución de los pacientes en un periodo determinado, difícilmente se podrá inferir de forma taxativa que el trabajo terapéutico llevado a cabo durante la estancia en el hospital haya podido influir en los resultados que hemos mencionado. Sin embargo, a partir de los resultados obtenidos podemos constatar las siguientes mejoras:

- Se reduce a la mitad el número de pacientes que precisan tratamiento farmacológico en el momento del alta clínica, respecto al inicio del tratamiento en el Hospital de Día. Además, dicha mejora se mantiene a lo largo del año de seguimiento e incluso sigue disminuyendo ligeramente.

- Disminución del grupo de chicos y chicas que «no estudian ni trabajan». Se trata de adolescentes que, en general, por su aislamiento y dificultades de relación se quedan «encerrados en casa», sin actividad alguna.

- Destacar, finalmente, que un 50\% de los adolescentes manifiestan -en la valoración subjetiva- la remisión total de los síntomas entre los 6 meses y el año, y que el $38 \%$ considera haber mejorado clínicamente.

\section{Conclusiones}

Con las limitaciones mencionadas podríamos decir que la mayor parte de los adolescentes con los cuales un año después se pudo contactar presentan, en sentido global, una evolución clínica satisfactoria. Señalar, asimismo, que un porcentaje de ellos además de no experimentar mejoría $(8,3 \%)$, continuaban con tratamiento farmacológico $(21,6 \%)$ y en cuanto a las relaciones familiares seguían siendo conflictivas o muy conflictivas $(8 \%)$. 
A partir de estos resultados nos planteamos si los pacientes con el diagnóstico de mayor gravedad (trastorno mental grave) son los que confirman la peor evolución. En este sentido insistir una vez más en la conveniencia de este tipo de trabajos, pues, a pesar del tiempo transcurrido en que fue formulado, «el dilema» (9) sería cómo poder ayudar mejor a aquellos chicos y chicas cuyas dificultades no son sólo las propias de la crisis de la adolescencia, sino las de una enfermedad que en esta etapa y, probablemente, a lo largo de su vida les seguirá acompañando.

\section{BIBLIOGRAFÍA}

(1) CRAF, M., «Psychiatric Day Hospitals», American Journal of Psychiatry, 1959, 116, pp. 251-254.

(2) GonçAlves, P., «Introduction et rappel historique», Bulletin Institutions Universitaires de Psychiatrie Genève (IUPG), 1994, 145, 5, Spécial Hôpitaux de jour.

(3) Bleandonu, G.; Despinoy, M., Hôpitaux de jour et psychiatrie dans la communauté, París, Payot, 1974.

(4) Blinder, B. J., y otros, «The Children's Psychiatric Hospital Unit in the Community: I. Concept and Development», Am. J. Psychiatry, 1978, 135, pp. 848-851.

(5) JiMÉnez PASCUAL, A. M., «Hospital de día psiquiátrico para niños y adolescentes», Revista de la A.E.N., 2001, XXI (77), pp. 115-124.

(6) JIMÉnez PASCUAL, A. M., «Dispositivos de internamiento terapéutico para patología mental grave de niños y adolescentes», Revista de la A.E.N., 2004, XXIII (89), pp. 167-186.

(7) ÉTUDES ET RECHERCHES SUR L'ENFANCE, Les hôpitaux de jour et externats psychothérapiques pour enfants, Toulouse, Privat, 1969, p. 72.

(8) Pascual, J. C., y otros, «A Naturalistic Study: 100 Consecutive Episodes of Acute Agitation in a Psychiatric Emergency Department», Actas Españolas de Psiquiatría, 2006, 34 (4), pp. 239-244.

(9) Masterson, J. F., El dilema psiquiátrico del adolescente, Buenos Aires, Paidós, 1972.

(10) SEMPERE, J., «El grupo multifamiliar en un hospital de día para adolescentes», Revista de Psicopatología y salud mental del niño y del adolescente, 2005, 5, pp. 93-101.

(11) Sebestik, J.; GarRalda, M. E., «Survey of Difficult to Contein and Treta Children and Adolescents», Archives of Disease In Childhood, 1996, 75 (1), pp. 78-81.

(12) Horvitz-Lennon, M., y otros, «Partial Versus Full Hospitalisation for Adults in Psychiatric Distress: a Systematic Review of the Published Literature (1957-1997)», American Journal of Psychiatry, 2001, 158 (5), pp. 676-85.

* Rubén D. Gualtero, sociólogo y coordinador del Dpto. de Investigación y Publicaciones de la Fundació Orienta; Mercè Gibert, médico psiquiatra, Jefe de Servicio de Hospitalización parcial de la Fundació Orienta; Ferran Cuerva, trabajador social y psicólogo; Marta Gomà, psicóloga (CSMIJ Sant Boi de Llobregat), Dpto. de Investigación y Publicaciones de la Fundació Orienta. Correspondencia: Dra. Mercè Gibert. Hospital de día para adolescentes de Gavà. Calle Sant Lluis, 64 - 08850 Gavà (Barcelona); M.Gibert@ fundacioorienta.com

** Fecha de recepción: 12-XII-2006. 